\title{
Distribution of serotypes and antibiotic resistance of invasive Pseudomonas aeruginosa in a multi-country collection
}

Shamima Nasrin 1,2, Nicolas Hegerle1,2, Shaichi Sen 1,2, Joseph Nkeze 1,2, Sunil Sen 1,2, Jasnehta Permala-Booth 1,2, Myeongjin Choi ${ }^{1,2}$, James Sinclair ${ }^{1,2}$, Milagritos D. Tapia 1,3, J. Kristie Johnson ${ }^{4}$, Samba O. Sow ${ }^{5}$, Joshua T. Thaden ${ }^{6}$, Vance G. Fowler $\mathrm{Jr}^{7,8}$, Karen A. Krogfelt ${ }^{9,10}$, Annelie Brauner ${ }^{11}$, Efthymia Protonotariou ${ }^{12}$, Eirini Christaki ${ }^{13,14}$, Yuichiro Shindo ${ }^{15}$, Andrea L. Kwa ${ }^{16,17,18}$, Sadia Shakoor ${ }^{19}$, Ashika Singh-Moodley ${ }^{20}$, Olga Perovic ${ }^{20}$, Jan Jacobs ${ }^{21,22}$, Octavie Lunguya ${ }^{23,24}$, Raphael Simon ${ }^{1,2}$, Alan S. Cross ${ }^{1,2+}$ and Sharon M. Tennant ${ }^{1,2^{*}}$

\begin{abstract}
Background: Pseudomonas aeruginosa is an opportunistic pathogen that causes a wide range of acute and chronic infections and is frequently associated with healthcare-associated infections. Because of its ability to rapidly acquire resistance to antibiotics, P. aeruginosa infections are difficult to treat. Alternative strategies, such as a vaccine, are needed to prevent infections. We collected a total of 413 P. aeruginosa isolates from the blood and cerebrospinal fluid of patients from 10 countries located on 4 continents during 2005-2017 and characterized these isolates to inform vaccine development efforts. We determined the diversity and distribution of $\mathrm{O}$ antigen and flagellin types and antibiotic susceptibility of the invasive $P$. aeruginosa. We used an antibody-based agglutination assay and PCR for $O$ antigen typing and PCR for flagellin typing. We determined antibiotic susceptibility using the Kirby-Bauer disk diffusion method.
\end{abstract}

Results: Of the 413 isolates, 314 (95\%) were typed by an antibody-based agglutination assay or PCR $(n=99)$. Among the 20 serotypes of $\mathrm{P}$. aeruginosa, the most common serotypes were $\mathrm{O} 1, \mathrm{O} 2, \mathrm{O} 3, \mathrm{O} 4, \mathrm{O}$, O6, O8, O9, $\mathrm{O} 10$ and $\mathrm{O} 11$; a vaccine that targets these 10 serotypes would confer protection against more than $80 \%$ of invasive $P$. aeruginosa infections. The most common flagellin type among 386 isolates was FlaB (41\%). Resistance to aztreonam (56\%) was most common, followed by levofloxacin (42\%). We also found that 22\% of strains were non-susceptible to meropenem and piperacillin-tazobactam. Ninety-nine (27\%) of our collected isolates were resistant to multiple antibiotics. Isolates with FlaA2 flagellin were more commonly multidrug resistant $(p=0.04)$.

Conclusions: Vaccines targeting common $\mathrm{O}$ antigens and two flagellin antigens, FlaB and FlaA2, would offer an excellent strategy to prevent $P$. aeruginosa invasive infections.

Keywords: Pseudomonas, Serotype, Flagellin, Multidrug resistance

*Correspondence: stennant@som.umaryland.edu

${ }^{+}$Alan S. Cross and Sharon M. Tennant co-senior authors; these authors contributed equally to this work.

${ }^{1}$ Center for Vaccine Development and Global Health, University of Maryland School of Medicine, 685 W. Baltimore St. - HSF1 Room 480, Baltimore, MD 21201, USA

Full list of author information is available at the end of the article

\section{Background}

Pseudomonas aeruginosa is an opportunistic human pathogen that causes acute life-threatening infections in elderly, critically ill and immunocompromised patients worldwide [1]. P. aeruginosa is a common cause of healthcare-associated infections (HAIs). Magill et al. [2] 
reported a point prevalence study in the United States which determined that $P$. aeruginosa was responsible for $7.1 \%$ of HAIs. This finding is supported by recent data from the National Healthcare Safety Network (NHSN) from 2015 to 2017 which reported $P$. aeruginosa as the fourth (8\%) most frequently isolated pathogen in all HAIs in the U.S. [3]. Likewise, in the European Union, Walter et al. [4] performed a point prevalence study to investigate healthcare-associated pneumonia and determined that $P$. aeruginosa was the most commonly isolated pathogen (17\%). This bacterium also causes chronic pulmonary infections in chronic wounds and cystic fibrosis (CF) patients [5, 6].

Severe life-threatening infections caused by $P$. aeruginosa are difficult to treat because of the organism's limited susceptibility to antimicrobial agents [7]. Multi-drug resistant (MDR) P. aeruginosa is a serious concern for hospitalized patients. The SENTRY antimicrobial surveillance program, which collects data from 400 medical centers worldwide, showed that most of the $P$. aeruginosa isolates were from patients with pneumonia followed by bloodstream infections during 1997-2016 [8]. The SENTRY surveillance program also reported that 27.7 and $23.7 \%$ of $P$. aeruginosa from pneumonia and bloodstream infections, respectively, were MDR [8]. MDR isolates were most frequently isolated from Latin America followed by Europe, North America and AsiaPacific. Unfortunately, no sites from Africa are included in the SENTRY program. The emergence of antibiotic resistance significantly limits therapeutic options for this bacterium.

O-polysaccharide (OPS), the most variable region of lipopolysaccharide (LPS), plays an important role in virulence and is responsible for conferring serogroup specificity [9]. Several serotyping systems have been proposed by various investigators; however, the most common serotyping scheme is the International Antigenic Typing Scheme (IATS). According to the IATS, $P$. aeruginosa have been classified into 20 different serotypes (O1 to $\mathrm{O} 20$ ) based on the structure of their O-polysaccharide $[10,11]$.

Studies have shown that isolates belonging to serotypes $\mathrm{O} 1, \mathrm{O} 6, \mathrm{O} 11$, and $\mathrm{O} 12$ account for $>65 \%$ of the $P$. aeruginosa infections $[12,13]$ and serotype $\mathrm{O} 4$ and $\mathrm{O} 12$ isolates are more frequently associated with resistance to various classes of antibiotics $[14,15]$. Serotyping data indicates that $P$. aeruginosa isolates of serotypes $\mathrm{O} 5, \mathrm{O} 6$ and $\mathrm{O} 11$ are prevalent in burn wound infections [16] and $\mathrm{O} 6$ and $\mathrm{O} 11$ are the most prevalent serotypes in pneumonia $[17,18]$. A study conducted at a Canadian hospital in 1992-1993 has found that O1, O3, O5, O6, O10, and $\mathrm{O} 11$ were the most frequently identified serotypes isolated from pus, urine, sputa and other sources [19].
Recently, in silico serotyping of $>1000$ genomes of $P$. aeruginosa strains using whole genome sequencing revealed that $70 \%$ of the isolates were $\mathrm{O} 3, \mathrm{O} 6, \mathrm{O} 11$ or $\mathrm{O} 12$ [20]. Donta et al. [21] reported that serotypes O1, O2/O5, O3, O4, O6, O7, O10 and O16, represent $90 \%$ of bacteremic $P$. aeruginosa strains. Data describing the distribution of O serotypes among invasive $P$. aeruginosa from diverse geographical locations is lacking.

A study conducted by Lu et al. [17] showed a relationship between clinical outcome of pneumonia and $P$. aeruginosa serotypes. Serotype O1 was associated with mortality and serotypes O6 and O11 were prevalent in critically ill patients [17]. Previous studies also showed that some serotypes are more virulent than others; for instance, serotype O11 clinical isolates were found to secrete exotoxin U (ExoU), a toxin of the Type III Secretion System, more frequently than other serotypes [18] and in a murine model of pneumonia, serotype $\mathrm{O} 11$ was associated with increased lung injury [22].

The flagellum of $P$. aeruginosa consists of a polymer of flagellin (Fla) protein encoded by the $\mathrm{fliC}$ gene of serotype A (including sub-type A1 and A2) or B. There is no evidence of switching between serotype A and type B flagellin for any particular $P$. aeruginosa strain [23]. Antibodies to the flagellin of $P$. aeruginosa are protective which has been documented in pre-clinical models of subcutaneous infections, burn wounds and pneumonia [24]. It is not known whether there is a relationship between strains of particular flagellin types and antimicrobial susceptibility.

To control the spread of $P$. aeruginosa infection, an effective vaccine or other alternative immunotherapy is urgently needed. Despite decades-long efforts by the scientific community, no vaccine has yet been licensed to prevent infections by this pathogen [25]. Several groups have developed vaccines to prevent $P$. aeruginosa infections by targeting lipopolysaccharide (LPS), flagella, alginate, outer membrane proteins (OMPs) and proteins of the Type III Secretion System (T3SS) [26-31]. A double-blind randomized Phase 3 trial in cystic fibrosis patients with a bivalent $P$. aeruginosa $\mathrm{A} / \mathrm{B}$ flagellin vaccine revealed robust and durable antibody titers, and modest but statistically significant protection against P. aeruginosa infection in cystic fibrosis patients [32]. Unfortunately, the company that manufactured this vaccine stopped production and it is not currently available, but these data establish the proof of concept that flagellin-based vaccines are efficacious. High molecular weight OPS have been used to develop a polysaccharide-based vaccine against $P$. aeruginosa [33]. We have extended this approach and created a vaccine using the novel Multiple Antigen Presenting System (MAPS) to target 8 P. aeruginosa O-serotypes $(\mathrm{O} 1, \mathrm{O} 2, \mathrm{O} 3, \mathrm{O} 4, \mathrm{O} 5, \mathrm{O} 6, \mathrm{O} 10$ and O11) [34]. 
In this study, we examined the diversity of O-antigen serotypes and flagellin types among invasive $P$. aeruginosa isolates to get better insight into globally circulating serotypes and their antibiotic susceptibility. The findings from the present study provide information about the global serotype distribution of this pathogen which will facilitate development of an effective vaccine against $P$. aeruginosa.

\section{Results}

\section{Antibiotic resistance}

In this study, we collected 413 invasive clinical isolates of $P$. aeruginosa from patients' blood and cerebrospinal fluid from 10 countries (Table 1). Antibiotic susceptibility was determined for 370 P. aeruginosa invasive isolates by the disk diffusion method. Isolates obtained from Greece were excluded from all analyses involved in antimicrobial resistance because they were part of an MDR collection (Supplementary Fig. S1 and Fig. S2). Invasive $P$. aeruginosa strains were most frequently resistant to the antibiotic aztreonam (56\%), followed by levofloxacin $42 \%$ (Table 2). We found $22 \%$ of strains were non-susceptible to carbapenems. The distribution of MDR $P$. aeruginosa varied by country. No MDR $P$. aeruginosa isolates were identified in Mali.

\section{Serotyping and global distribution of invasive $P$. aeruginosa}

All $413 P$. aeruginosa invasive isolates were serotyped using a commercially available antibody-based serotyping kit (Supplementary Fig. S1). The distribution of serotypes is shown in Fig. 1A. The most prevalent serotypes were $\mathrm{O} 11(n=89 ; 22 \%), \mathrm{O} 1(n=58 ; 14 \%)$ and $\mathrm{O} 6$ $(n=53 ; 13 \%)$ which accounted for around $50 \%$ of the
P. aeruginosa strains that are circulating worldwide. Ninety-nine isolates (24\%) were non-typable using the commercially available antibody-based serotyping kit. To serotype these non-typable isolates $(n=99)$, we used an O-antigen specific PCR (Fig. 1B). Unlike the antibody-based agglutination assay which can serotype $P$. aeruginosa strains into $17 \mathrm{O}$ types, PCR can categorize isolates into 11 groups of gene clusters that are highly divergent from one another at the DNA sequence level [35]. The most prevalent serotype identified using PCR was $\mathrm{O} 6$ followed by the $\mathrm{O} 2 / \mathrm{O} 5 / \mathrm{O} 16 / \mathrm{O} 18 / \mathrm{O} 20$ cluster which comprised 9 and $6 \%$ of the isolates, respectively. We were able to serotype $95 \%(n=393)$ of the $P$. aeruginosa isolates using both antibody-based agglutination and PCR methods. Only $5 \%(n=20)$ of the isolates remained non-typable after employing both serotyping methods. We randomly selected 8 isolates that did not agglutinate with typing antibodies but were identified as $\mathrm{O} 1, \mathrm{O} 2 / \mathrm{O} 5 / \mathrm{O} 16 / \mathrm{O} 18 / \mathrm{O} 20, \mathrm{O} 3 / \mathrm{O} 15$ or O6 strains by PCR and showed that they were able to produce O-side chains of the LPS (Supplementary Fig. S3). We have determined the serotype of $95 \%$ of the $P$. aeruginosa isolates using agglutination and PCR methods (Fig. 1C). Taken together, we found that the most common $\mathrm{O}$ serotypes amongst 413 strains include O1, O2/O5/ O16/O18/O20, O3/O15, O6, O7/O8, O9, O10/O19 and O11/O17 which represented $92 \%$ of the isolates.

We also determined the global serotype distribution of $P$. aeruginosa isolates among different countries using the agglutination data (Fig. 2). Overall, we observed diverse serotypes of invasive $P$. aeruginosa circulating worldwide. Clinically important O1, O6 and $\mathrm{O} 11$ serotypes were generally the most common in each country.

Table 1 Source and origin of Pseudomonas aeruginosa isolates used in this study

\begin{tabular}{|c|c|c|c|c|c|}
\hline Region & Country & Institute of isolation & Source & Years of isolation & No. of strains \\
\hline \multirow[t]{2}{*}{ North America } & USA & University of Maryland, Medical Center & Blood & 2010 to 2015 & 116 \\
\hline & & Duke University Medical Center & Blood & 2012 to 2015 & 51 \\
\hline \multirow[t]{3}{*}{ Europe } & Sweden & Karolinska University Hospital & Blood & 2015 & 25 \\
\hline & Greece & AHEPA University Hospital & Blood & 2012 to 2016 & 43 \\
\hline & Denmark & Statens Serum Institute & Blood & 2017 & 35 \\
\hline \multirow[t]{3}{*}{ Africa } & South Africa & NICD a South Africa & Blood & 2015 & 50 \\
\hline & Mali & $C V D^{b}-M a l i$ & Blood/CSF & 2005 to 2012 & 24 \\
\hline & DR Congo & ITM Belgium & Blood & 2015 & 3 \\
\hline \multirow[t]{3}{*}{ Asia } & Japan & Nagoya University Hospital & Blood & 2010 and 2015 & 14 \\
\hline & Singapore & Singapore General Hospital & Blood & 2012 to 2016 & 25 \\
\hline & Pakistan & Aga Khan University & Blood & 2015 to 2017 & 27 \\
\hline
\end{tabular}

\footnotetext{
a National Institute for Communicable Diseases

${ }^{\mathrm{b}}$ Center for Vaccine Development and Global Health

${ }^{c}$ Institute of Tropical Medicine, Antwerp
} 
Table 2 Antibiotic resistance of invasive P. aeruginosa

\begin{tabular}{|c|c|c|c|c|c|c|c|c|c|}
\hline \multirow[t]{3}{*}{ Country $^{a}$} & \multirow{3}{*}{$\begin{array}{l}\text { No. of } \\
\text { isolates }\end{array}$} & \multicolumn{7}{|c|}{ No. of isolates (\%) non-susceptible to } & \multirow[t]{3}{*}{ MDR } \\
\hline & & \multicolumn{2}{|c|}{ Aminoglycoside } & \multirow{2}{*}{$\begin{array}{l}\text { Carbapenem } \\
\text { Meropenem }\end{array}$} & \multirow{2}{*}{$\begin{array}{l}\text { Cephalosporin } \\
\text { Cefepime }\end{array}$} & \multirow{2}{*}{$\begin{array}{l}\text { Fluoroquinolone } \\
\text { Levofloxacin }\end{array}$} & \multirow{2}{*}{$\begin{array}{l}\beta \text {-lactamase } \\
\text { inhibitor } \\
\text { Piperacillin- } \\
\text { tazobactam }\end{array}$} & \multirow{2}{*}{$\begin{array}{l}\text { Monobactam } \\
\text { Aztreonam }\end{array}$} & \\
\hline & & Amikacin & Gentamicin & & & & & & \\
\hline USA ${ }^{b}$ & 116 & $0(0)$ & $10(8.6)$ & $23(19.8)$ & $10(8.6)$ & $48(41.4)$ & $24(21.0)$ & $56(48.3)$ & $28(24.1)$ \\
\hline$U_{S A}{ }^{c}$ & 51 & $0(0)$ & $7(14.0)$ & $9(17.6)$ & $5(10.0)$ & $21(41.2)$ & $13(25.5)$ & $27(53.0)$ & $14(27.4)$ \\
\hline Denmark & 35 & $2(5.7)$ & $2(5.7)$ & $6(17.1)$ & $5(14.3)$ & $20(57.1)$ & $5(14.3)$ & $27(77.1)$ & $8(22.8)$ \\
\hline Sweden & 25 & $2(8.0)$ & $2(8.0)$ & $4(16.0)$ & $2(8.0)$ & $9(36.0)$ & $3(12.0)$ & $6(24.0)$ & $5(20.0)$ \\
\hline $\begin{array}{l}\text { South } \\
\text { Africa }\end{array}$ & 50 & $8(16.0)$ & $15(30.0)$ & $18(36.0)$ & $17(34.0)$ & $25(50.0)$ & $15(30.0)$ & $42(84.0)$ & $20(40.0)$ \\
\hline Mali & 24 & $1(4.2)$ & $1(4.2)$ & $0(0)$ & $0(0)$ & $1(4.2)$ & $0(0)$ & $8(33.3)$ & $0(0)$ \\
\hline DR Congo & 3 & $0(0)$ & $2(66.7)$ & $1(33.3)$ & $1(33.3)$ & $2(66.7)$ & $2(66.7)$ & $2(66.7)$ & $2(66.7)$ \\
\hline Japan & 14 & $0(0)$ & $0(0)$ & $4(28.6)$ & $0(0)$ & 4 (28.6) & $0(0)$ & $7(50.0)$ & $2(14.3)$ \\
\hline Singapore & 25 & $2(8.0)$ & $7(28.0)$ & $9(36.0)$ & $9(36.0)$ & $12(48.0)$ & $10(40.0)$ & $16(64.0)$ & $11(44.0)$ \\
\hline Pakistan & 27 & $6(22.2)$ & $6(22.2)$ & $8(29.6)$ & $8(29.6)$ & $14(51.8)$ & $9(33.3)$ & $15(55.5)$ & $9(33.3)$ \\
\hline Overall & 370 & $21(5.7)$ & $52(14.0)$ & $82(22.2)$ & $57(15.4)$ & $156(42.2)$ & $81(22.0)$ & $206(55.7)$ & $99(26.7)$ \\
\hline
\end{tabular}

${ }^{a}$ Isolates collected from Greece were excluded

${ }^{\mathrm{b}}$ Isolates were obtained from University of Maryland Medical Center

${ }^{c}$ Isolates were obtained from Duke University Medical Center

\section{Flagellin typing of $P$. aeruginosa}

The most common flagellin type among 386 (flagellin typing was not performed on 27 isolates due to logistic constraints) invasive $P$. aeruginosa isolates was FlaB (41\%), followed by FlaA2 (31\%) and FlaA1 (28\%) (Fig. 3A). No PCR product was observed for three strains (one from Singapore which was not motile and two from Pakistan which were motile). We found that FlaB was prevalent in isolates from South Africa (58\%), Pakistan (52\%) and Greece (51\%) whereas isolates from Denmark and Sweden were predominantly FlaA2 (49\%) and FlaA1 (48\%) (Fig. 3B). Overall, we observed diverse profiles of flagellin types among different countries. We observed a significant association between $\mathrm{O}$ serotypes and flagellin type (Chi-squared test, $p<0.001$ ) where strains that possess FlaA1 were likely to be associated with serotype O6 (38.3\%) and FlaA2 isolates were likely to be serotype $\mathrm{O} 11$ (42.4\%) or O1 (22.9\%) (Fig. 3C). Isolates with FlaB were likely to be associated with serotype O11 (15.9\%) and O3 (14.6\%). Furthermore, non-typable O serotypes were associated with FlaA1 and FlaB.

To determine if particular flagellar types were associated with antibiotic resistance, we evaluated the distribution of flagellar types among susceptible and non-susceptible strains for each antibiotic and for MDR and non-MDR strains. We observed that FlaA2 was significantly $(p<0.05)$ associated with MDR. In contrast, FlaB was more common amongst non-MDR than MDR strains $(p<0.05)$ (Fig. 4A). The percentage of flagellar types in susceptible and non-susceptible groups to each antibiotic is shown in Fig. 4B. We found significantly more FlaA2 isolates that were non-susceptible to gentamicin, levofloxacin, piperacillin-tazobactam and aztreonam compared to susceptible $(p<0.05)$. In contrast, FlaB type was significantly more common in isolates susceptible to meropenem, cefepime, levofloxacin and piperacillin-tazobactam. We did not find any significant difference in FlaA1 distribution in susceptible versus non-susceptible strains.

\section{Discussion}

In this study, we collected more than 400 invasive $P$. aeruginosa isolates from geographically diverse locations to determine their serotype distribution, flagellin types and antibiotic susceptibility pattern to understand the epidemiology of this pathogen and to guide vaccine development efforts. The challenges for developing an effective vaccine against $P$. aeruginosa are multifactorial, interplaying between the pathogen's virulence factors, ability to utilize multiple pathways to cause infection, antibiotic resistance, emerging high-risk clones and host factors [36]. Emergence of MDR P. aeruginosa is a serious public health concern.

We observed that $26.7 \%$ of the $P$. aeruginosa isolates tested $(N=370)$ were MDR. Our data are similar to those of the SENTRY surveillance program which found $23.7 \%$ MDR out of 52,022 P. aeruginosa isolates collected in 1997-2016 from > 400 medical centers representing 

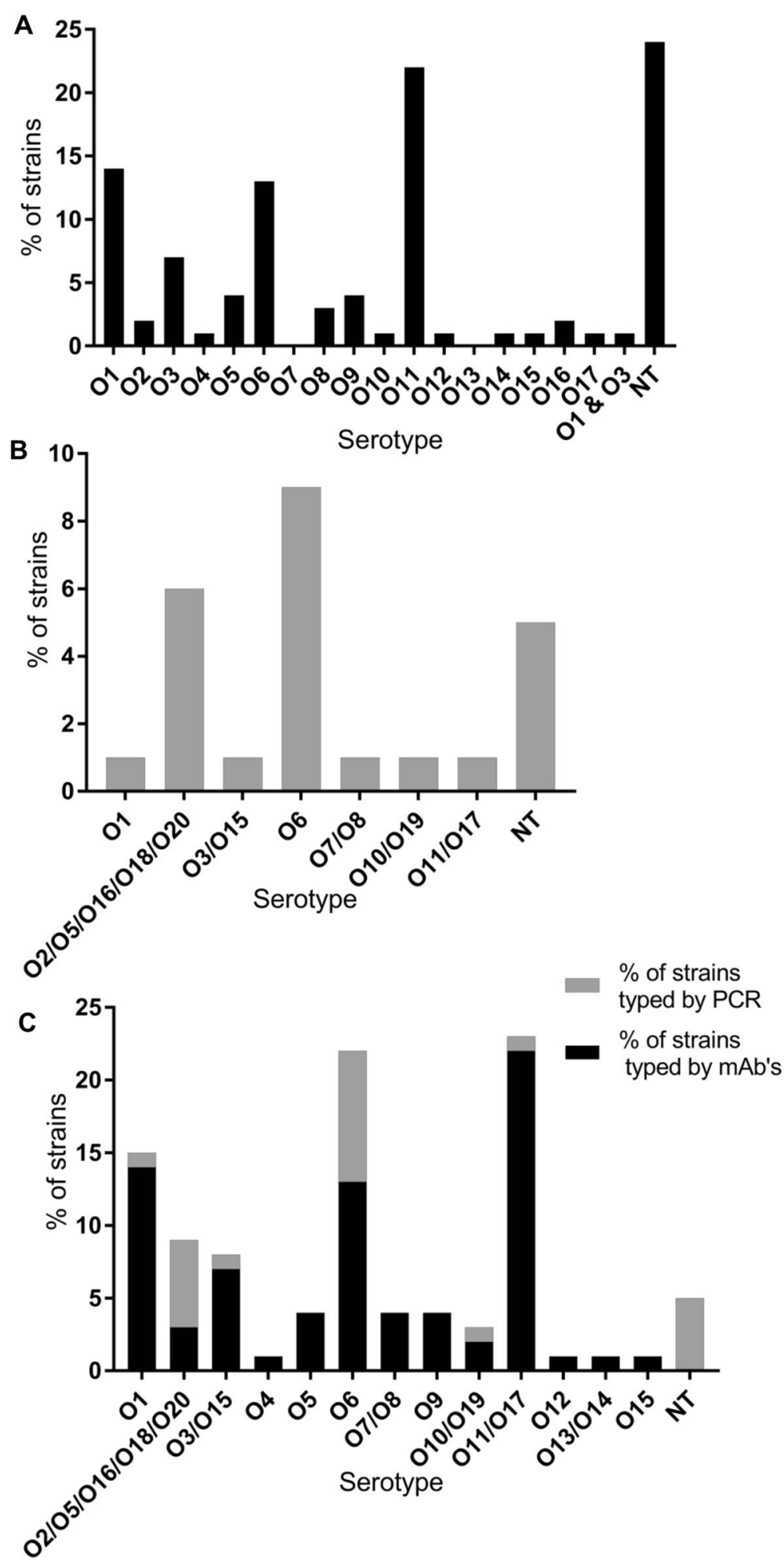

Fig. 1 Distribution of O serotypes in 413 invasive P. aeruginosa isolates. A O serotypes were determined by slide agglutination assay. B Isolates that were non-typable (NT) by the slide agglutination assay were O-typed by PCR $(n=99)$. C Distribution of O serotypes using slide agglutination and PCR assays for O-antigen typing. mAb's, monoclonal antibodies 


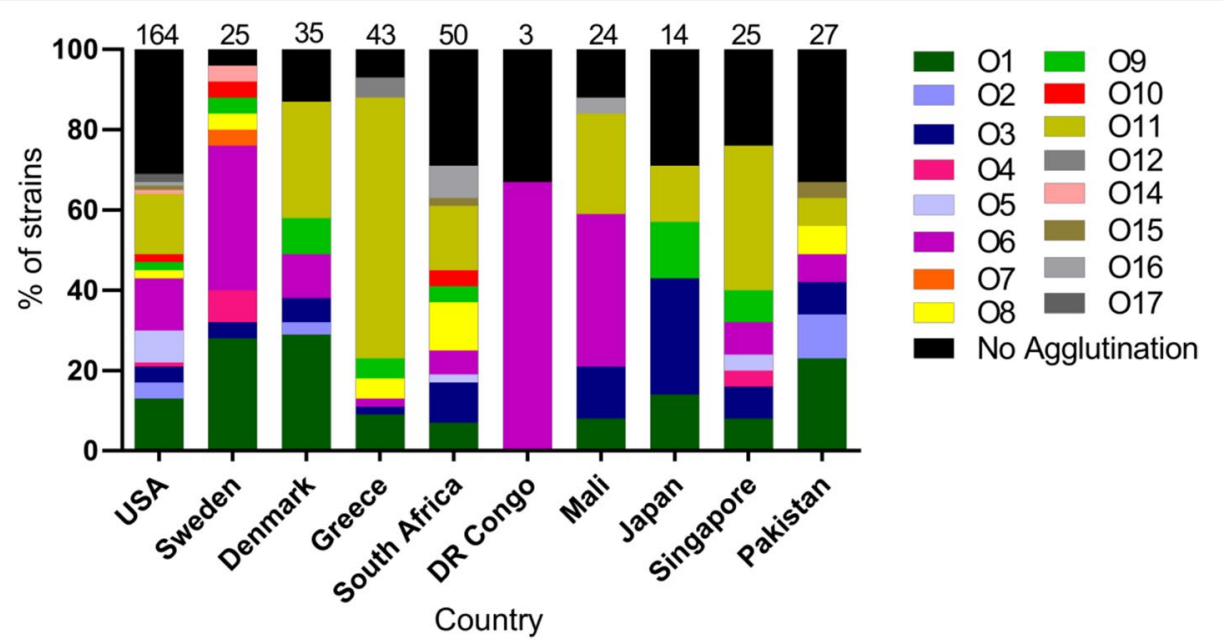

Fig. 2 Diversity of $P$. aeruginosa O serotypes by country using an antibody based serotyping kit. The numbers on top of the graph represent the number of isolates tested from each country

Asia-Pacific, Europe, Latin America and North America [8]. We found the highest prevalence of MDR isolates from Singapore, South Africa and Democratic Republic of the Congo (albeit only 3 isolates of the latter were tested).

Cefepime is a widely used fourth generation cephalosporin that has potent antimicrobial activity against Pseudomonas [37]. However, resistance of $P$. aeruginosa to cefepime has been observed in several other studies [37-39] as well as in this study. This observation of resistance to cefepime is of concern as Akhabue et al. [38] showed a significant association between infections caused by invasive cefepime-resistant $P$. aeruginosa and increased mortality among hospitalized patients.

In our study, $22 \%$ of the $P$. aeruginosa were resistant to meropenem, an antibiotic that has been considered as the most effective agent for the treatment of $P$. aeruginosa infections [40]. Our observations are similar to that of the SENTRY surveillance program which reported that $22.3 \%$ of bloodstream isolates were resistant to meropenem [8]. In contrast, other studies have reported higher resistance to carbapenems; among $2070 P$. aeruginosa isolates from 27 hospitals located in 14 European and Mediterranean countries, $94.5 \%$ were resistant to imipenem, $81.6 \%$ to meropenem and $57.3 \%$ to doripenem using Clinical and Laboratory Standards Institute (CLSI) breakpoints [41]. The increased prevalence of carbapenem resistance among the 2070 P. aeruginosa isolates reported by Castanheira et al. might be due to the varied source of the isolates which were obtained from bloodstream, nosocomial respiratory tract infections, skin, urine and other unknown sources (in contrast to our study where isolates were obtained from blood or cerebrospinal fluid). It is worth noting that the World Health Organization (WHO) recently ranked carbapenem-resistant $P$. aeruginosa as critical in their global priority pathogens list of MDR bacteria to help in prioritizing the research and development of new and effective antibiotic treatments [42].

One of the objectives of this study was to identify the most common serotypes of $P$. aeruginosa circulating to inform development of O-polysaccharide-based vaccines. We found that the distribution of serotypes varied amongst investigation sites and countries, but the most prevalent serotypes were $\mathrm{O} 1, \mathrm{O} 6$ and $\mathrm{O} 11$, which comprise about $50 \%$ of tested isolates. These results are concordant with findings from previous studies [13]. To our knowledge, this is the first study to evaluate the distribution of $P$. aeruginosa serotypes in invasive infections from multiple (ten) countries with diverse geographical locations. Our antibody-based serotyping data indicated that $>70 \%$ of the isolates belonged to 10 serotypes $(\mathrm{O} 1, \mathrm{O} 2, \mathrm{O} 3, \mathrm{O} 4, \mathrm{O} 5, \mathrm{O} 6, \mathrm{O} 8, \mathrm{O} 9, \mathrm{O} 10$ and O11). However, antibody-based serotyping data in combination with the molecular method, determined that $>80 \%$ of the strains belonged to these 10 serotypes. Thus, targeting these 10 serotypes in a vaccine could conceivably confer protection against infection caused by a large number of invasive $P$. aeruginosa strains. We and others have observed a low prevalence of serotype O12 in clinical strains [13, 18]; however, other studies have shown that isolates of serotype $\mathrm{O} 12$ are more frequently associated with antibiotic resistance [14]. Therefore, serotype $\mathrm{O} 12$ might be included in future vaccine programs to prevent spread of antimicrobial resistant strains. 

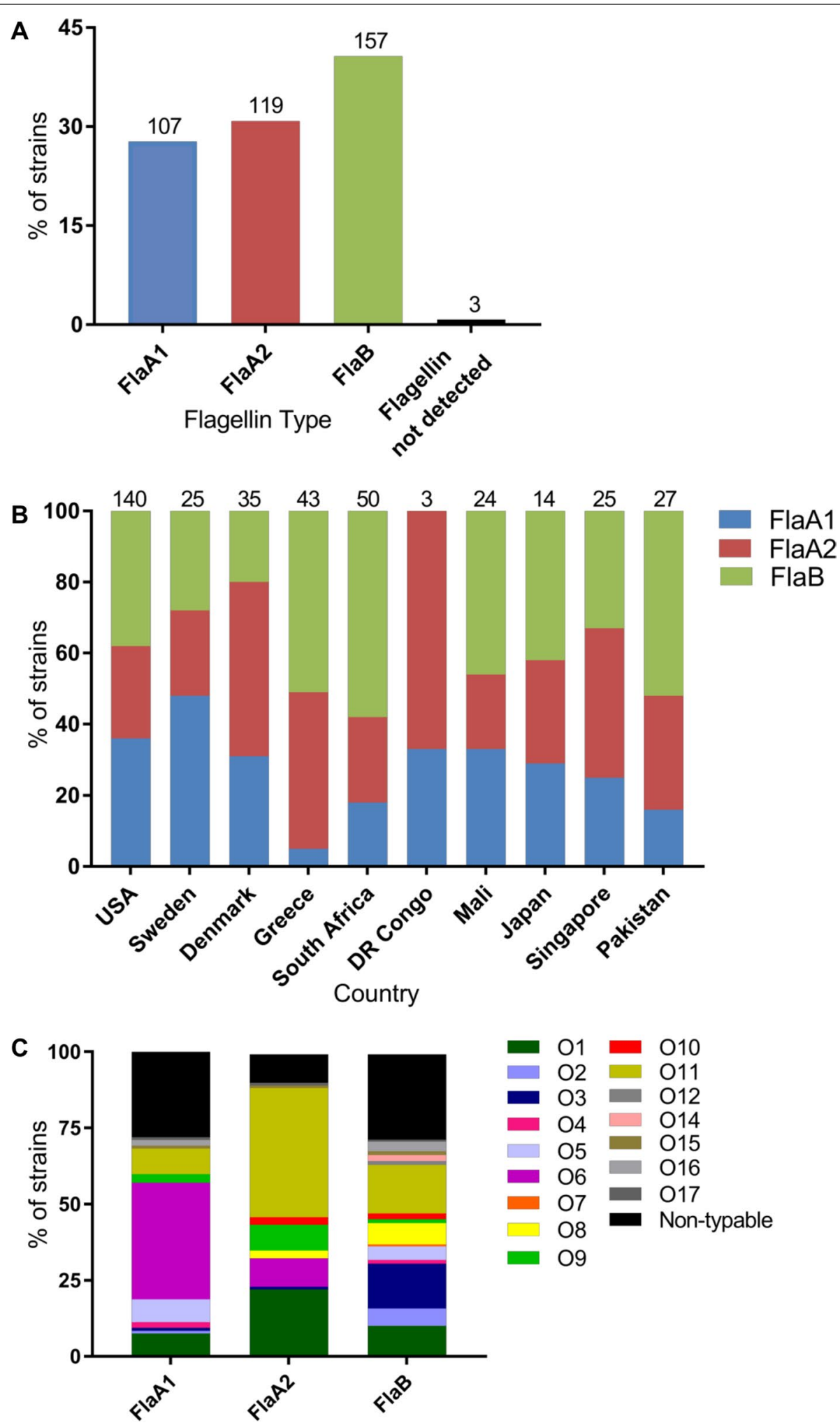

Flagellin Type

Fig. 3 Distribution of flagellin types. A Distribution of flagellin types amongst 386 invasive P. aeruginosa isolates by PCR. The numbers on top of the graph represent the number of isolates of each flagellin type. B Distribution of flagellin types by country. The numbers on top of the graph represent the number of isolates tested from each country. $\mathbf{C}$ Distribution of $\mathrm{O}$ serotypes by flagellin type 


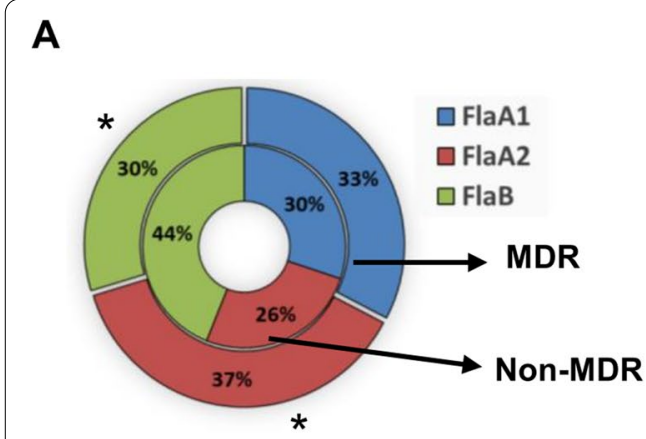

B

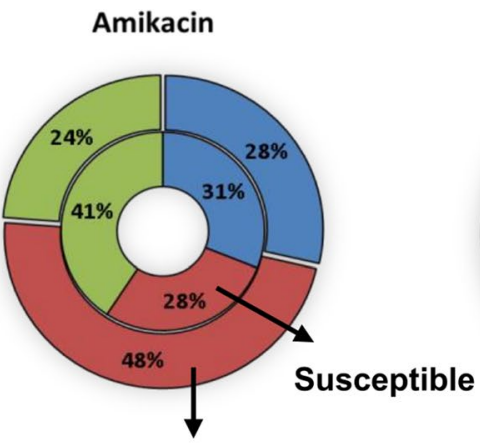

Non-susceptible

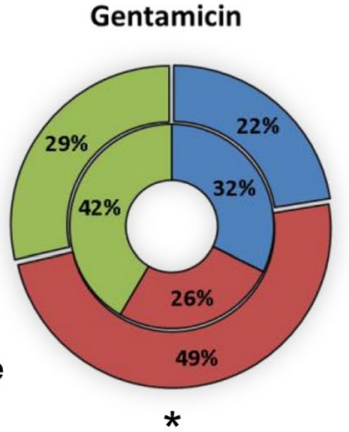

*
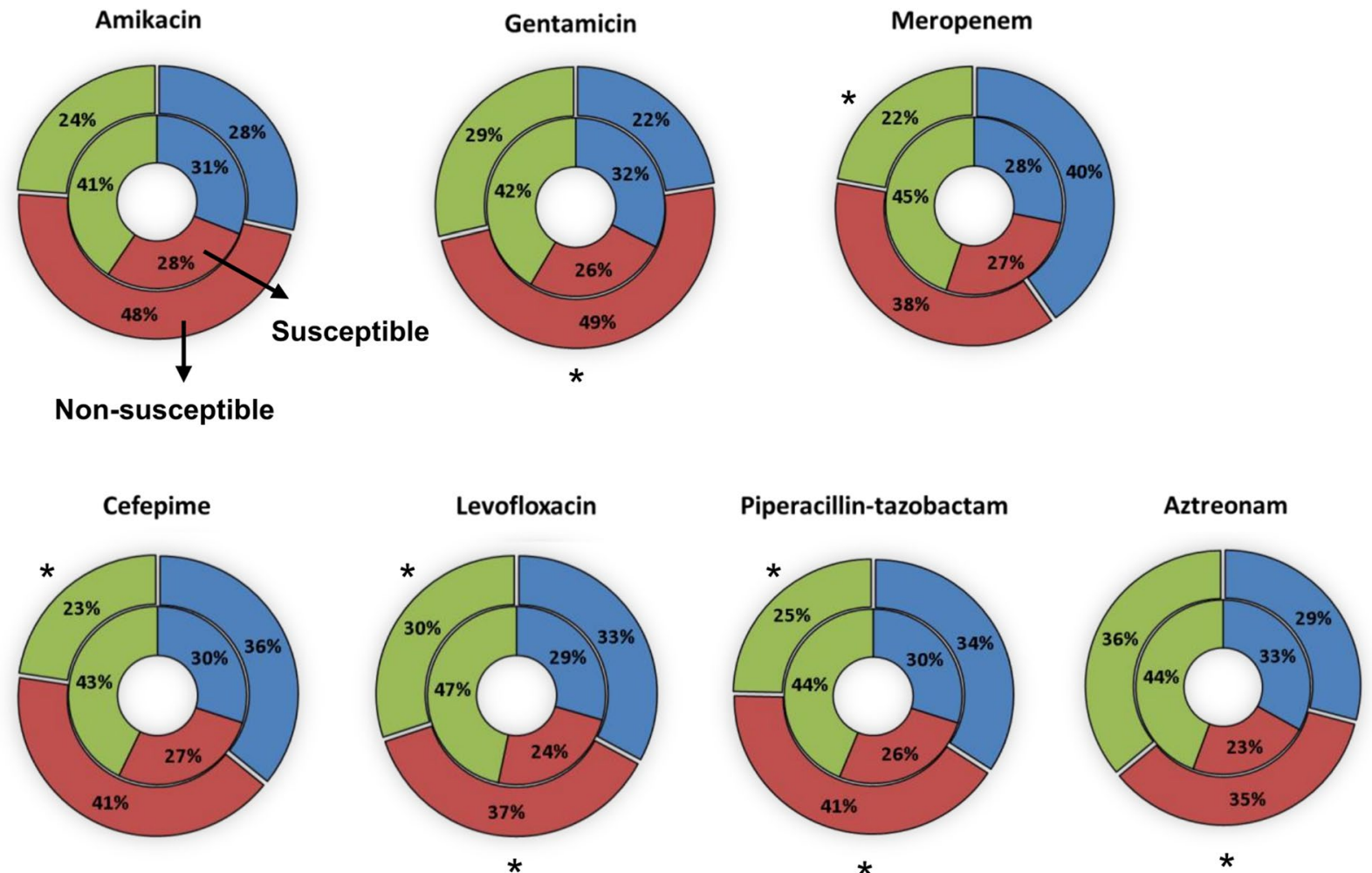

Fig. 4 Association between flagellin types and antibiotic resistance in clinical P. aeruginosa strains. A Inner circle of the pie represents the percentage of flagellin types that are non-MDR and the outer circle of the pie represents the percentage of flagellin types that are MDR. B The percentage of flagellin types in susceptible isolates (inner circle) and in non-susceptible isolates (outer circle) to each antibiotic. Statistically significant differences between MDR and non-MDR and susceptible and non-susceptible groups are indicated by asterisks $\left({ }^{*}\right)$. Isolates obtained from Greece were excluded from the analysis

In the current study, the most common flagellin type was FlaB (40.6\%) followed by FlaA2 (30.8\%) and FlaA1 (27.7\%) and distribution of flagellin types among countries was diverse. We found that strains with FlaA1 were significantly associated with $\mathrm{O} 6$ and O11; FlaA2 strains were frequently serotyped as $\mathrm{O} 1$ or $\mathrm{O} 11$; and isolates that possessed $\mathrm{FlaB}$ were significantly associated with $\mathrm{O} 11$ and O3. We also observed that FlaA2 was the predominant flagellin type among MDR $P$. aeruginosa strains and was associated with resistance to gentamicin, levofloxacin, piperacillin-tazobactam and aztreonam. The role of flagella or flagellin proteins in virulence of $P$. aeruginosa have been previously documented [43]; however, to our knowledge, no association between flagellin type and antibiotic resistance has been described for P. aeruginosa. Given the conserved nature and limited flagellin types in $P$. aeruginosa, it would be worthwhile to include flagellin proteins as a vaccine component to improve vaccine 
coverage. Our data suggests that although FlaB was the most common flagellin type, it would also be important to target FlaA2 strains which are associated with antibiotic resistance.

\section{Conclusions}

We determined antibiotic susceptibility of invasive $P$. aeruginosa collected from 10 countries located on 4 different continents. The distribution of MDR $P$. aeruginosa varied by country. $P$. aeruginosa strains were most frequently resistant to the antibiotic aztreonam, followed by levofloxacin and $22 \%$ of the invasive isolates were nonsusceptible to carbapenems. Our data suggest that a multivalent vaccine that targets $10 \mathrm{O}$ serotypes $(\mathrm{O} 1, \mathrm{O} 2, \mathrm{O} 3$, O4, O5, O6, O8, O9, O10 and O11) would confer protection against $>80 \%$ of circulating invasive $P$. aeruginosa isolates. We also determined that if one was to include flagellin antigens in a vaccine, it would be important to include FlaB (most common flagellin type) and FlaA2 (associated with antibiotic resistance). The findings from this study will help to guide efforts to develop an effective vaccine against invasive $P$. aeruginosa.

\section{Materials and methods}

\section{Bacterial isolates collection and growth conditions}

Four hundred and thirteen invasive clinical isolates of $P$. aeruginosa were collected during 2005-2017 from ten countries located in four different geographic regions (Table 1). Bacterial isolates were collected from patients' blood and cerebrospinal fluid. As reference strains, the International Antigenic Typing Scheme (IATS) serotypetype specific strains, IATS O1, O2, O3, O4, O5, O6, O7, O8, O9, O10, O11, O12, O13, O14, O15, O16, O17, were used. P. aeruginosa was isolated and identified at each site using standard microbiologic protocols. As needed, identity was confirmed by morphological characteristics and biochemical profile using API 20NE (bioMérieux, Durham, NC) at the Center for Vaccine Development and Global Health (CVD), University of Maryland, Baltimore, Maryland. All confirmed $P$. aeruginosa isolates were preserved in Tryptic Soy Broth (TSB) supplemented with $15 \%\left(\mathrm{vol} / \mathrm{vol}\right.$ ) glycerol and stored at $-80^{\circ} \mathrm{C}$ for future use. When needed, bacteria were streaked for single colonies on animal product-free Hy-Soy agar plates $(0.5 \%$ sodium chloride, $1 \%$ animal free soytone [TEKNova, Hollister, CA], 0.5\% Hy-Yest 444 [Kerry Biosciences, Beloit, WI]). For routine culture, cells were incubated at $37^{\circ} \mathrm{C}$ for $18-24 \mathrm{~h}$ unless otherwise indicated. For motility tests, bacteria were stab inoculated using a sterile pipette tip onto a motility medium containing $1 \%$ tryptone, $0.5 \%$ $\mathrm{NaCl}$, and $0.4 \%$ agar and the plates were incubated for $18 \mathrm{~h}$ at $37^{\circ} \mathrm{C}$.

\section{Antibiotic susceptibility testing}

Antibiotic susceptibility studies were performed by Kirby-Bauer disk diffusion method according to CLSI guidelines (M100-S29). The following antibiotics were tested in this study: amikacin $(30 \mu \mathrm{g})$, aztreonam $(30 \mu \mathrm{g})$, cefepime $(30 \mu \mathrm{g})$, gentamicin $(10 \mu \mathrm{g})$, meropenem $(10 \mu \mathrm{g})$, piperacillin-tazobactam $(110 \mu \mathrm{g})$ and levofloxacin $(5 \mu \mathrm{g})$. Cartridges of antibiotic impregnated discs were purchased from Becton Dickinson Company Ltd. (Franklin Lakes, NJ). Antibiotic susceptibility was determined by measuring the diameter of the zone of inhibition and the results were interpreted as susceptible (S), intermediate (I) and resistant (R) based on CLSI guidelines [44]. The criterion used in this study for defining multidrugresistant (MDR) in $P$. aeruginosa was defined as nonsusceptibility (including both resistant and intermediate) to three or more classes of antibiotics [45]. In this study, non-MDR isolates were defined as those susceptible to all antibiotics or resistant to 1 or 2 classes of antibiotics.

\section{O-antigen serotyping}

$P$. aeruginosa isolates were serotyped by slide agglutination using commercially available antibodies specific to $P$. aeruginosa (MediMabs, Montreal, Canada) according to the manufacturer's instructions. Briefly, $P$. aeruginosa was streaked onto Hy-Soy agar medium and incubated for $16-18 \mathrm{~h}$ at $37^{\circ} \mathrm{C}$. After incubation, cells were serotyped using three groups of pooled antisera (pool A, pool $\mathrm{B}$ and pool C) followed by a panel of 17 monovalent antisera $(\mathrm{O} 1, \mathrm{O} 2, \mathrm{O} 3, \mathrm{O} 4, \mathrm{O} 5, \mathrm{O} 6, \mathrm{O} 7, \mathrm{O} 8, \mathrm{O} 9, \mathrm{O} 10, \mathrm{O} 11$, O12, O13, O14, O15, O16 and O17) based on the IATS serotyping system. Cells producing clear aggregation and clumping in the presence of antibody were considered as serotype-positive, whereas cells that remained uniformly suspended were considered as serotype-negative.

Isolates which were non-typable by slide agglutination assay were $\mathrm{O}$-serotyped by PCR according to a method described previously [35]. Genomic DNA was extracted from each of the non-typable isolates using the GenElute genomic DNA purification kit (Sigma-Aldrich, Billerica, MA) according to the manufacturer's instructions. $\mathrm{O}$-antigen specific PCR was performed as follows: each $25 \mu \mathrm{l}$ reaction mixture contained $2.5 \mu \mathrm{l}$ of $10 \times$ Greentaq buffer (Genscript, Piscataway, NJ), $0.5 \mu \mathrm{l}$ of $10 \mu \mathrm{M}$ deoxynucleotide triphosphate mix (dNTPs) (Genscript), $1.0 \mu \mathrm{l}$ of $10 \mu \mathrm{M}$ forward and reverse primer mix (See Table S1 for details of primers), 0.5 U of Greentaq DNA polymerase (Genscript) and $40 \mathrm{ng}$ of DNA template. Cycling parameters were $95^{\circ} \mathrm{C}$ for $3 \mathrm{~min}$ followed by 35 cycles of $95^{\circ} \mathrm{C}$ for $30 \mathrm{~s}, 60^{\circ} \mathrm{C}$ for $30 \mathrm{~s}$ and $72^{\circ} \mathrm{C}$ for $45 \mathrm{~s}$ to $1.5 \mathrm{~min}$ (depending on the primer mixes) with a final extension at $72^{\circ} \mathrm{C}$ for $5 \mathrm{~min}$. PCR amplicons $(10 \mu \mathrm{l})$ were 
electrophoresed on 1.0\% agarose gels and photographed using a ChemiDoc ${ }^{\mathrm{TM}}$ MP imaging system (Bio-Rad, Hercules, CA). O-serotype was determined based on the presence or absence of an amplicon.

\section{Lipopolysaccharide (LPS) extraction}

Eight representative non-typable $P$. aeruginosa isolates were selected to confirm expression of $\mathrm{O}$ polysaccharide. Crude LPS was extracted from $P$. aeruginosa isolates, electrophoresed and visualized by staining. P. aeruginosa strain PAK was used as a positive control. Cells were grown overnight in $3 \mathrm{ml}$ of Hy-Soy broth in a shaking incubator at $37^{\circ} \mathrm{C}$ and $200 \mathrm{rpm}$. After incubation, cells were pelleted by centrifugation followed by washing the pellet twice with $1 \times$ PBS to remove any pyocyanin from the media (to remove pigments that can affect spectrophotometric measurements). Pellets were resuspended in PBS and optical density (OD) at $600 \mathrm{~nm}$ was adjusted to 1.0. One milliliter of the $\mathrm{OD}_{600}$ standardized culture was centrifuged at $15,600 \times g$ for $1 \mathrm{~min}$. Supernatant was discarded carefully without disturbing the pellet. The pellet was suspended in $250 \mu \mathrm{l}$ of Laemmli buffer $1 \times$ with $\beta$-mercaptoethanol (BME). Samples were boiled at $100^{\circ} \mathrm{C}$ for $20 \mathrm{~min}$, allowed to cool at room temperature and then $20 \mu \mathrm{g}$ of proteinase K (Qiagen Germantown, MD) was added to the solution. The solution was incubated at $55^{\circ} \mathrm{C}$ for $4 \mathrm{~h}$. Proteinase $\mathrm{K}$ was then heat inactivated at $100^{\circ} \mathrm{C}$ for $10 \mathrm{~min}$. For electrophoresis, $25 \mu \mathrm{l}$ of the sample and $2 \mu \mathrm{l}$ of CandyCane glycoprotein molecular weight standard (Invitrogen Waltham, MA) was loaded onto a NuPAGE 4-12\% Bis-Tris Gel (Invitrogen Carlsbad, $\mathrm{CA})$ and LPS visualized using the Pro- $\mathrm{Q}^{\circledR}$ Emerald 300 lipopolysaccharide gel stain kit according to the manufacturer's instructions (Thermo Fisher Waltham, MA).

\section{Flagellin typing of Pseudomonas aeruginosa}

To determine flagellin types for $P$. aeruginosa isolates, the central region of the $f l i C$ gene was amplified by PCR and sequenced using primer pair CW46_F (5'-GGC CTGCAGATCNCCAA-3') and CW45_R (5'-GGCAGC TGGTTNGCCTG-3') [46]. The primer pair used in this study produced amplicons that can differentiate between A and B type flagellin. Boiled lysates of $P$. aeruginosa isolates were used as DNA template for performing flagellin-specific PCR. Four to six colonies were suspended in $150 \mu \mathrm{l}$ of nuclease free water. Samples were boiled at $100^{\circ} \mathrm{C}$ for $10 \mathrm{~min}$ and then pelleted by centrifugation at $14,000 \mathrm{rpm}$ for $5 \mathrm{~min}$. The supernatants were collected and used as DNA template. PCR was performed as follows: each $50 \mu \mathrm{l}$ reaction mixture contained $5.0 \mu \mathrm{l}$ of $10 \times$ Greentaq buffer (Genscript), $1.5 \mu \mathrm{l}$ of $2 \mathrm{mM}$ deoxynucleotide triphosphate mix (dNTPs) (Genscript), $3.0 \mu \mathrm{l}$ of $10.0 \mu \mathrm{M}$ forward and reverse primer mix, $1.0 \mathrm{U}$ of
Greentaq DNA polymerase (Genscript) and $1.0 \mu \mathrm{l}$ of DNA template. Reactions were incubated at $95^{\circ} \mathrm{C}$ for $3 \mathrm{~min}$ followed by 30 cycles of denaturation at $95^{\circ} \mathrm{C}$ for $30 \mathrm{~s}$, annealing at $56^{\circ} \mathrm{C}$ for $45 \mathrm{~s}$, and extension at $72^{\circ} \mathrm{C}$ for $1.5 \mathrm{~min}$, with a final extension at $72^{\circ} \mathrm{C}$ for $5 \mathrm{~min}$. The PCR products were analyzed by agarose gel electrophoresis. To differentiate between $\mathrm{A} 1$ and $\mathrm{A} 2$ subtypes, a new set of primers (PA_FLIC_F: 5'-ATGGCCTTGACCGTC AAC- $3^{\prime}$ and PA_FLIC_R: 5'-GCGCAGCAGGCTCAG AAC-3') were used to generate $f l i C$ amplicons and for sequencing. PCR was performed according to the conditions described above.

Amplicons were then purified using the Qiagen PCR purification kit (Germantown, MD) according to the manufacturer's protocol. Purified PCR products were sequenced by Genewiz (South Plainfield, NJ). Sequences were de novo assembled and compared with reference sequences using the Geneious software package.

\section{Statistical analysis}

The proportion of susceptible versus resistant strains for each flagellin type was analyzed by Chi-squared analysis with Yate's correction using GraphPad Prism version 7 software (GraphPad Software, San Diego). The significance level was $p<0.05$. The association between O serotype and flagellin type was determined by Chi-squared test using Stata/SE version 16 software.

\section{Supplementary Information}

The online version contains supplementary material available at https://doi. org/10.1186/s12866-021-02427-4.

Additional file 1: Table S1. Primers used for $O$ antigen serotyping. Figure $\mathbf{S 1}$. The number of $P$. aeruginosa isolates tested by each method. *Flagellin typing was not performed on 27 isolates from Duke University due to logistic constraints. Figure $\mathbf{S 2}$. Antibiotic resistance of isolates from Greece. Figure S3. The O-antigen profiles of representative non-typable $P$. aeruginosa isolates determined by SDS-PAGE. LPS from eight non-typable P.aeruginosa strains were extracted and separated on a 4-12\% Bis-Tris SDS-polyacrylamide gel and stained with Pro-Q Emerald 300 according to manufacturer's instructions. CandyCane glycoprotein molecular weight standard (ladder), P. aeruginosa strain PAK (+ve control).

\section{Acknowledgments}

We thank Dr. Joseph Lam, University of Guelph, Ontario, Canada, for the kind gift of IATS typing strains. We thank Ghazala Shaheen and Humaira Shafaq from Departments of Pathology and Pediatrics, the Aga Khan University, Karachi, Pakistan; Lemonia Skoura at Department of Microbiology, AHEPA University Hospital, Greece; Marie-France Phoba from Department of Clinical Microbiology, National Institute for Biomedical Research, and Department of Microbiology, University Hospital of Kinshasa, Democratic Republic of the Congo; Barbara Barbé at Department of Clinical Sciences, Institute of Tropical Medicine, Antwerp, Belgium; Tze-Peng Lim at Department of Pharmacy, Singapore General Hospital, Singapore, Singapore and Emerging Infectious Diseases, Duke-National University of Singapore Graduate Medical School, Singapore, Singapore; Mamadou Sylla, Adama Mamby Keita, Boubou Tamboura and Aliou Toure from Centre pour le Développement des Vaccins, Mali, Bamako, Mali; Karen L. Kotloff from Center for Vaccine Development and Global Health (CVD) and Department of Pediatrics, University of Maryland 
School of Medicine, Baltimore, MD, USA. We thank Yuanyuan Liang, Department of Epidemiology and Public Health at University of Maryland School of Medicine for advice on statistical analyses.

\section{Authors' contributions}

Conception of the study: RS, ASC and SMT. Design of the study: RS, ASC and SMT. Acquisition or analysis of data: SN, NH, SS, JN, SS, JP-B, MC, JS, MDT, JKJ, SOS, JTT, VGF Jr., KAK, AB, EP, EC, YS, ALK, SS, AS-M, OP, JJ and OL. Interpretation of data: SN, JP-B, ASC and SMT. Wrote the original draft of the manuscript: SN. Substantively revised the manuscript: ASC and SMT. The author(s) read and approved the final manuscript.

\section{Funding}

This work was funded by Nosocomial Vaccine Corporation. The funder had no role in the design of the study and collection, analysis, and interpretation of data and in writing the manuscript.

\section{Availability of data and materials}

All data generated or analyzed during this study are included in this published article (and its supplementary information files). DNA sequences used to determine the flagellin type are available in GenBank (accession numbers OL354146 - OL354417).

\section{Declarations}

\section{Ethics approval and consent to participate}

No human data were used in this study. Bacterial isolates were de-identified. This study was designated as non-human research by the University of Maryland Baltimore Institutional Review Board.

\section{Consent for publication}

Not applicable.

\section{Competing interests}

SMT, RS and ASC are holders of US patents 9,988,426 and 10,556,931 titled "Broad spectrum conjugate vaccine to prevent Klebsiella pneumoniae and Pseudomonas aeruginosa infections." All other authors declare no competing interests.

\section{Author details}

${ }^{1}$ Center for Vaccine Development and Global Health, University of Maryland School of Medicine, 685 W. Baltimore St. - HSF1 Room 480, Baltimore, MD 21201, USA. ${ }^{2}$ Department of Medicine, University of Maryland School of Medicine, Baltimore, MD, USA. ${ }^{3}$ Department of Pediatrics, University of Maryland School of Medicine, Baltimore, MD, USA. ${ }^{4}$ Department of Pathology, University of Maryland School of Medicine, Baltimore, MD, USA. ${ }^{5}$ Centre pour le Développement des Vaccins, Mali, Bamako, Mali. ${ }^{6}$ Division of Infectious Diseases, Duke University Medical Center, Durham, NC, USA. ${ }^{7}$ Division of Infectious Diseases and International Health, Department of Medicine, Duke University School of Medicine, Durham, NC, USA. ${ }^{8}$ Duke Clinical Research Institute, Durham, NC, USA. ${ }^{9}$ Statens Serum Institut, Copenhagen, Denmark. ${ }^{10}$ Department of Natural Sciences and Environment, Roskilde University, Roskilde, Denmark. ${ }^{11}$ Department of Microbiology, Tumor and Cell Biology, Division of Clinical Microbiology, Karolinska Institutet and Karolinska University Hospital, 17176 Stockholm, Sweden. ${ }^{12}$ Department of Microbiology, AHEPA University Hospital, Thessaloniki, Greece. ${ }^{13}$ Department of Medicine, AHEPA University Hospital, Thessaloniki, Greece. ${ }^{14}$ University of Cyprus Medical School, Nicosia, Cyprus. ${ }^{15}$ Department of Respiratory Medicine, Nagoya University Graduate School of Medicine, Nagoya, Japan. ${ }^{16}$ Department of Pharmacy, Singapore General Hospital, Singapore, Singapore. ${ }^{17}$ Emerging Infectious Diseases, Duke-National University of Singapore Medical School, Singapore, Singapore. ${ }^{18}$ Department of Pharmacy, Faculty of Science, National University of Singapore, Singapore, Singapore. ${ }^{19}$ Departments of Pathology and Pediatrics, Aga Khan University, Karachi, Pakistan. ${ }^{20}$ National Institute for Communicable Diseases a Division of the National Health Laboratory Service, and School of Pathology, Faculty of Health Sciences, University of the Witwatersrand, Johannesburg, South Africa. ${ }^{21}$ Department of Clinical Sciences, Institute of Tropical Medicine, Antwerp, Belgium. ${ }^{22}$ Department of Microbiology, Immunology and Transplantation, KU Leuven, Leuven, Belgium. ${ }^{23}$ Department of Clinical Microbiology, National Institute for Biomedical Research, Kinshasa,
Democratic Republic of the Congo. ${ }^{24}$ Department of Microbiology, University Hospital of Kinshasa, Kinshasa, Democratic Republic of the Congo.

Received: 3 June 2021 Accepted: 6 December 2021

Published online: 06 January 2022

\section{References}

1. Sadikot RT, Blackwell TS, Christman JW, Prince AS. Pathogen-host interactions in Pseudomonas aeruginosa pneumonia. Am J Respir Crit Care Med. 2005;171:1209-23.

2. Magill SS, Edwards JR, Bamberg W, Beldavs ZG, Dumyati G, Kainer MA, et al. Multistate point-prevalence survey of health care-associated infections. N Engl J Med. 2014;370:1198-208.

3. Weiner-Lastinger LM, Abner S, Edwards JR, Kallen AJ, Karlsson M, Magill SS, et al. Antimicrobial-resistant pathogens associated with adult healthcare-associated infections: summary of data reported to the National Healthcare Safety Network, 2015-2017. Infect Control Hosp Epidemiol. 2020:41:1-18.

4. Walter J, Haller S, Quinten C, Kärki T, Zacher B, Eckmanns T, et al. Healthcare-associated pneumonia in acute care hospitals in European Union/European Economic Area countries: an analysis of data from a point prevalence survey, 2011 to 2012. Euro Surveill. 2018;23:1700843.

5. Waters CM, Goldberg JB. Pseudomonas aeruginosa in cystic fibrosis: a chronic cheater. Proc Natl Acad Sci U S A. 2019;116:6525-7.

6. Malhotra S, Hayes D Jr, Wozniak DJ. Cystic fibrosis and Pseudomonas aeruginosa: the host-microbe interface. Clin Microbiol Rev. 2019;32:e00138-18.

7. Carmeli Y, Troillet N, Eliopoulos GM, Samore MH. Emergence of antibioticresistant Pseudomonas aeruginosa: comparison of risks associated with different antipseudomonal agents. Antimicrob Agents Chemother. 1999:43:1379-82.

8. Shortridge D, Gales AC, Streit JM, Huband MD, Tsakris A, Jones RN. Geographic and temporal patterns of antimicrobial resistance in Pseudomonas aeruginosa over 20 years from the SENTRY antimicrobial surveillance program, 1997-2016. Open Forum Infect Dis. 2019;6:S63-S8.

9. Stanislavsky ES, Lam JS. Pseudomonas aeruginosa antigens as potential vaccines. FEMS Microbiol Rev. 1997;21:243-77.

10. Liu PV, Matsumoto H, Kusama H, Bergan T. Survey of heat-stable, major somatic antigens of Pseudomonas aeruginosa. Int J Syst Bacteriol. 1983;33:256-64.

11. Liu PV, Wang S. Three new major somatic antigens of Pseudomonas aeruginosa. J Clin Microbiol. 1990;28:922-5.

12. Pirnay JP, Biloca F, Pot B, Cornelis P, Zizi M, Van Eldere J, et al. Pseudomonas aeruginosa population structure revisited. PLoS One. 2009:4:e7740.

13. Bert F, Lambert-Zechovsky N. Comparative distribution of resistance patterns and serotypes in Pseudomonas aeruginosa isolates from intensive care units and other wards. J Antimicrob Chemother. 1996;37:809-13.

14. Thrane SW, Taylor VL, Freschi L, Kukavica-Ibrulj I, Boyle B, Laroche J, et al. The widespread multidrug-resistant serotype $\mathrm{O} 12$ Pseudomonas aeruginosa clone emerged through concomitant horizontal transfer of serotype antigen and antibiotic resistance gene clusters. mBio. 2015;6:e01396-15.

15. Del Barrio-Tofiño E, Sánchez-Diener I, Zamorano L, Cortes-Lara S, LópezCausapé C, Cabot G, et al. Association between Pseudomonas aeruginosa O-antigen serotypes, resistance profiles and high-risk clones: results from a Spanish nationwide survey. J Antimicrob Chemother. 2019;74:3217-20.

16. Estahbanati HK, Kashani PP, Ghanaatpisheh F. Frequency of Pseudomonas aeruginosa serotypes in burn wound infections and their resistance to antibiotics. Burns. 2002;28:340-8.

17. Lu Q, Eggimann P, Luyt C-E, Wolff M, Tamm M, François B, et al. Pseudomonas aeruginosa serotypes in nosocomial pneumonia: prevalence and clinical outcomes. Crit Care. 2014;18:R17-R.

18. Faure K, Shimabukuro D, Ajayi T, Allmond LR, Sawa T, Wiener-Kronish JP. O-antigen serotypes and type III secretory toxins in clinical isolates of Pseudomonas aeruginosa. J Clin Microbiol. 2003;41:2158-60.

19. Ravaoarinoro M, Mohapatra S, Shore J, Rawal S, Omri A, Yaghi J, et al. Serotyping clinical isolates of Pseudomonas aeruginosa in relation to infection site antibiotic susceptibility and beta-lactamase production. Int J Antimicrob Agents. 1996;7:65-8. 
20. Thrane SW, Taylor VL, Lund O, Lam JS, Jelsbak L. Application of wholegenome sequencing data for $\mathrm{O}$-specific antigen analysis and in silico serotyping of Pseudomonas aeruginosa isolates. J Clin Microbiol. 2016:54:1782-8

21. Donta ST, Peduzzi P, Cross AS, Sadoff J, Haakenson C, Cryz SJ Jr, et al. Immunoprophylaxis against Klebsiella and Pseudomonas aeruginosa infections. The federal Hyperimmune immunoglobulin trial study group. J Infect Dis. 1996;174:537-43.

22. Le Berre R, Nguyen S, Nowak E, Kipnis E, Pierre M, Quenee L, et al. Relative contribution of three main virulence factors in Pseudomonas aeruginosa pneumonia. Crit Care Med. 2011;39:2113-20.

23. Sabharwal N, Dhall S, Chhibber S, Harjai K. Molecular detection of virulence genes as markers in Pseudomonas aeruginosa isolated from urinary tract infections. Int J Mol Epidemiol Genet. 2014;5:125-34.

24. Döring G, Pier GB. Vaccines and immunotherapy against Pseudomonas aeruginosa. Vaccine. 2008;26:1011-24.

25. Vincent JL. Vaccine development and passive immunization for Pseudomonas aeruginosa in critically ill patients: a clinical update. Future Microbiol. 2014;9:457-63.

26. Alexander JW, Fisher MW, MacMillan BG. Immunological control of Pseudomonas infection in burn patients: a clinical evaluation. Arch Surg. 1971;102:31-5.

27. Rosok MJ, Stebbins MR, Connelly K, Lostrom ME, Siadak AW. Generation and characterization of murine antiflagellum monoclonal antibodies that are protective against lethal challenge with Pseudomonas aeruginosa. Infect Immun. 1990;58:3819-28.

28. Bartell PF, Orr TE, Chudio B. Purification and chemical composition of the protective slime antigen of Pseudomonas aeruginosa. Infect Immun. 1970;2:543-8

29. von Specht B, Knapp B, Hungerer K, Lücking C, Schmitt A, Domdey H. Outer membrane proteins of Pseudomonas aeruginosa as vaccine candidates. J Biotechnol. 1996;44:145-53.

30. Sato H, Frank DW. Multi-functional characteristics of the Pseudomonas aeruginosa type III needle-tip protein, PcrV; comparison to orthologs in other gram-negative bacteria. Front Microbiol. 2011;2:142.

31. Hegerle N, Choi M, Sinclair J, Amin MN, Ollivault-Shiflett M, Curtis B, et al. Development of a broad spectrum glycoconjugate vaccine to prevent wound and disseminated infections with Klebsiella pneumoniae and Pseudomonas aeruginosa. PLoS One. 2018;13:e0203143.

32. Döring G, Meisner C, Stern M. Flagella vaccine trial study G. a doubleblind randomized placebo-controlled phase III study of a Pseudomonas aeruginosa flagella vaccine in cystic fibrosis patients. Proc Natl Acad Sci U S A. 2007;104:11020-5.

33. Pier GB, Thomas DM. Characterization of the human immune response to a polysaccharide vaccine from Pseudomonas aeruginosa. J Infect Dis. 1983; 148:206-13.

34. Simon R. Novel multivalent vaccine for gram-negative bacterial pathogens including multiple antibiotic resistant strains: World Vaccine Congress, Washington DC; 2019.

35. Raymond CK, Sims EH, Kas A, Spencer DH, Kutyavin TV, Ivey RG, et al. Genetic variation at the O-antigen biosynthetic locus in Pseudomonas aeruginosa. J Bacteriol. 2002;184:3614.

36. Juan C, Peña C, Oliver A. Host and pathogen biomarkers for severe Pseudomonas aeruginosa infections. J Infect Dis. 2017;215:S44-s51.

37. Sader HS, Fritsche TR, Jones RN. Potency and spectrum trends for cefepime tested against 65746 clinical bacterial isolates collected in north American medical centers: results from the SENTRY antimicrobial surveillance program (1998-2003). Diagn Microbiol Infect Dis. 2005;52:265-73.

38. Akhabue E, Synnestvedt M, Weiner MG, Bilker WB, Lautenbach E. Cefepime-resistant Pseudomonas aeruginosa. Emerg Infect Dis. 2011;17:1037-43.

39. SuTY, Ye JJ, Hsu PC, Wu HF, Chia JH, Lee MH. Clinical characteristics and risk factors for mortality in cefepime-resistant Pseudomonas aeruginosa bacteremia. J Microbiol Immunol Infect. 2015;48:175-82.

40. Meletis G, Exindari M, Vavatsi N, Sofianou D, Diza E. Mechanisms responsible for the emergence of carbapenem resistance in Pseudomonas aeruginosa. Hippokratia. 2012;16:303-7.

41. Castanheira M, Deshpande LM, Costello A, Davies TA, Jones RN. Epidemiology and carbapenem resistance mechanisms of carbapenemnon-susceptible Pseudomonas aeruginosa collected during 2009-11 in
14 European and Mediterranean countries. J Antimicrob Chemother. 2014;69:1804-14.

42. Tacconelli E, Carrara E, Savoldi A, Harbarth S, Mendelson M, Monnet DL, et al. Discovery, research, and development of new antibiotics: the WHO priority list of antibiotic-resistant bacteria and tuberculosis. Lancet Infect Dis. 2018;18:318-27.

43. Feldman M, Bryan R, Rajan S, Scheffler L, Brunnert S, Tang H, et al. Role of flagella in pathogenesis of Pseudomonas aeruginosa pulmonary infection. Infect Immun. 1998;66:43.

44. CLSI. Performance standards for antimicrobial susceptibility testing. 30th ed. CLSI supplement M100. Wayne, PA: Clinical and Laboratory Standards Institute; 2020

45. Magiorakos AP, Srinivasan A, Carey RB, Carmeli Y, Falagas ME, Giske CG, et al. Multidrug-resistant, extensively drug-resistant and pandrugresistant bacteria: an international expert proposal for interim standard definitions for acquired resistance. Clin Microbiol Infect. 2012;18:268-81.

46. Campodónico VL, Llosa NJ, Grout M, Döring G, Maira-Litrán T, Pier GB. Evaluation of flagella and flagellin of Pseudomonas aeruginosa as vaccines. Infect Immun. 2010;78:746-55.

\section{Publisher's Note}

Springer Nature remains neutral with regard to jurisdictional claims in published maps and institutional affiliations.
Ready to submit your research? Choose BMC and benefit from:

- fast, convenient online submission

- thorough peer review by experienced researchers in your field

- rapid publication on acceptance

- support for research data, including large and complex data types

- gold Open Access which fosters wider collaboration and increased citations

- maximum visibility for your research: over $100 \mathrm{M}$ website views per year

At BMC, research is always in progress.

Learn more biomedcentral.com/submissions 